\title{
BMJ Open Effectiveness of omega-3 fatty acid administration on completion rate of adjuvant chemotherapy for biliary tract cancer: study protocol for a single- centre, open-label, single-arm, historically controlled study
}

Kimihiko Ueno, Tetsuo Ajiki, Daisuke Tsugawa, Masayuki Akita, Yu Hashimoto, Masahide Awazu, Hideyo Mukubo, Shohei Komatsu, Kaori Kuramitsu, Sachio Terai, Motofumi Tanaka, Hirichika Toyama, Masahiro Kido, Takumi Fukumoto

To cite: Ueno K, Ajiki T, Tsugawa D, et al. Effectiveness of omega-3 fatty acid administration on completion rate of adjuvant chemotherapy for biliary tract cancer: study protocol for a single-centre, openlabel, single-arm, historically controlled study. BMJ Open 2019;9:e029915. doi:10.1136/ bmjopen-2019-029915

- Prepublication history for this paper is available online. To view these files, please visit the journal online (http://dx.doi. org/10.1136/bmjopen-2019029915).

Received 19 February 2019 Revised 09 July 2019 Accepted 16 July 2019

D) Check for updates

(c) Author(s) (or their employer(s)) 2019. Re-use permitted under CC BY-NC. No commercial re-use. See rights and permissions. Published by BMJ.

Hepato-Biliary-Pancreatic Surgery, Kobe University, Kobe, Japan

Correspondence to

Kimihiko Ueno;

kueno.rhmn@gmail.com

\section{ABSTRACT}

Introduction Multimodal treatment prolongs the survival of patients with biliary tract cancer (BTC). However, the chemotherapy choices for this disease are few, and completing each chemotherapy session is important. Adjuvant chemotherapy has been attempted for BTC, but has only had a $75 \%$ completion rate. Body weight loss and cholangitis are reasons for the interruption of chemotherapy. Previous reports suggested that nutritional intervention with omega-3 fatty acids maintained body weight and improved the completion rate for chemotherapy. Moreover, omega- 3 fatty acids have an anti-inflammatory effect. Therefore, we theorised that omega-3 fatty acids would improve the completion rate of adjuvant chemotherapy in patients with BTC. The aim of this study is thus to evaluate the effectiveness of omega-3 fatty acids for patients planning adjuvant chemotherapy for BTC.

Method and analysis This study is a single-centre, open-label, single-arm, historically controlled study with a planned enrolment of 55 participants. Protocol treatment consists of four courses of S-1 adjuvant chemotherapy and an oral omega-3 fatty acid pharmaceutic adjuvant (LOTRIGA $2 \mathrm{~g}$ (Takeda Pharmaceutical Co.)), which includes $2 \mathrm{~g}$ of omega- 3 fatty acids from day 1 until day 168 of the treatment period. The primary endpoint is the completion rate of four total courses of S-1. Secondary endpoints are postoperative cholangitis, time to recurrence or distant metastasis, changes in nutritional index, changes in the lymphocyte blast transformation test induced by phytohaemagglutinin, and concanavalin A and diamine oxidase serum activity during adjuvant chemotherapy. All adverse events will be evaluated.

Ethics and dissemination This protocol was approved by the Institutional Review Board of Kobe University Hospital. The findings from this study will be presented at national and international conferences and published in peerreviewed journals.

Trial registration number UMIN000031247.
Strengths and limitations of this study

This study investigates the effectiveness of omega-3 fatty acids on the completion rate of adjuvant chemotherapy for biliary tract cancer (BTC).

- This study is well planned for the selection of participants with BTC based on four different cancers depending on location.

- A major limitation of this study is related to it being a historically controlled study, which includes some bias.

- Because the primary endpoint of this study is the completion rate of chemotherapy, not the prognosis of patients, the effect on survival can only be interpreted as a guide.

\section{INTRODUCTION}

While surgical resection is a definitive treatment for biliary tract cancer (BTC), the 5 -year survival rate is about $30 \%-50 \%$, which is lower than that of other gastrointestinal cancers. Because of the limitation of surgical resection, multimodal treatment is expected to improve rates of survival. ${ }^{2}$ In spite of the importance of adjuvant chemotherapy, the initiation and continuation of this in patients have proven difficult after biliary surgery. Biliary surgery, such as liver resection and pancreaticoduodenectomy, is very invasive, and easily results in delayed recovery as well as bacterial translocation (BT) that arises from malnutrition and immunological deterioration. Uncontrolled BT causes cholangitis that interrupts chemotherapy. A feasibility study of S-1 adjuvant chemotherapy for BTC showed that $75.8 \%$ of patients completed a 
24-week protocol. ${ }^{3}$ The main causes of discontinuation were adverse events and relapse. This rate is similar to that for gastric $(78 \%)^{4}$ and pancreatic $(72 \%)$ cancers. $^{5}$ Few chemotherapy choices for BTC exist and, therefore, the completion of each chemotherapy session is essential. It therefore follows that an improvement in the completion rate for adjuvant chemotherapy is important for the treatment of BTC.

Previous reports have suggested that body weight loss may be a reason for interruptions in adjuvant chemotherapy. ${ }^{6}$ Nutritional intervention with omega-3 fatty acids maintained body weight ${ }^{78}$ and improved the completion rate of adjuvant chemotherapy. ${ }^{7}$ Omega-3 fatty acids are metabolised to lipid mediators that evoke an anti-inflammatory response as well as novel proresolving mechanisms, and enhances microbial clearance. ${ }^{9}$ In addition, enteral immunonutrition using omega-3 fatty acids reduces BT and atrophy of intestinal mucosal villi in rats with obstructive jaundice. Omega-3 fatty acids are expected to be a nutritional intervention for patients after biliary surgery in terms of the maintenance of body weight and immunity. Moreover, since previous reports have suggested an anti-tumour effect for omega-3 fatty acids, ${ }^{10-13}$ this is an appropriate drug for cancer patients.

The aim of this study is to evaluate the efficacy of omega-3 fatty acids on adjuvant chemotherapy. Because patients to whom chemotherapy was administered tended to reduce their dietary intake, and those after biliary surgery are prone to cholangitis, omega-3 fatty acids are used in this study for nutritional support and as anti-inflammatory treatment. We will conduct this study with the hypothesis that omega-3 fatty acids improve tolerance for adjuvant chemotherapy after biliary surgery with regard to increasing body weight and reducing cholangitis.

\section{METHODS AND ANALYSIS \\ Study design}

This study is a single-centre, open-label, single-arm, historically controlled study of patients who are administered S-1 after surgical resection of BTC. The aim of this study is to examine any improvement in completion rates of adjuvant chemotherapy by the administration of omega- 3 fatty acids. All participants are scheduled to receive administration of four cycles of S-1 adjuvant chemotherapy, and oral omega-3 fatty acids during chemotherapy (figure 1). This study includes a 3-year period of registration and 1-year period of follow-up. This study protocol follows the Standard Protocol Items: Recommendations for Interventional Trials statement.

\section{SAMPLE SIZE}

The sample size was calculated based on the primary endpoint. This study is planned for 55 participants. A previous study reported that the percentage completion for four courses of S-1 chemotherapy was $76 \%$ in patients with BTC. ${ }^{3}$ The reasons for discontinuance were adverse

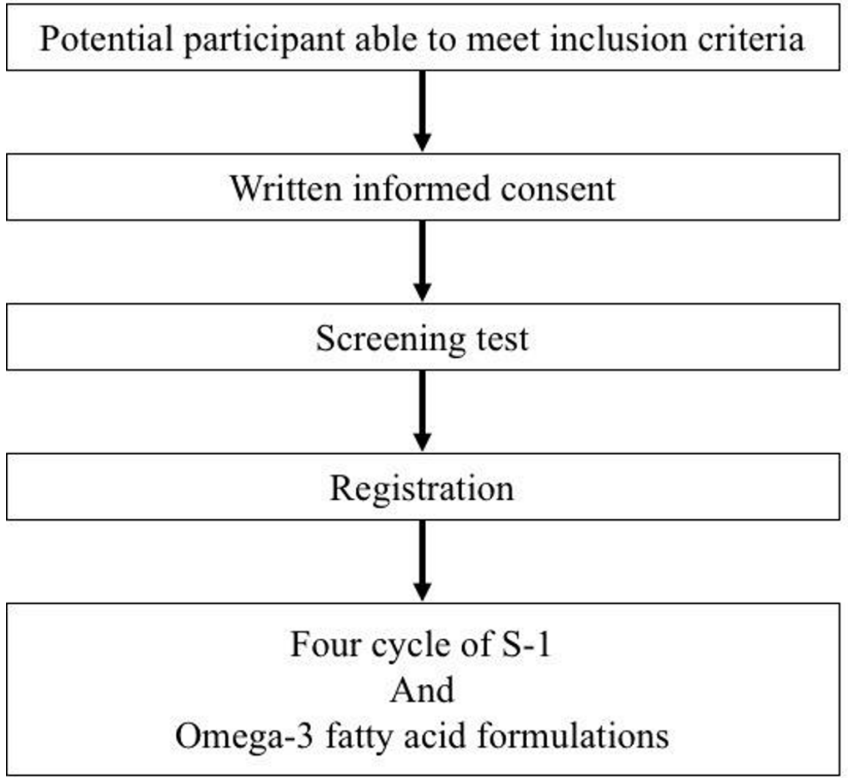

Figure 1 Summary of study.

events $(12 \%)$ and early recurrence $(12 \%)$. We set 0.76 as the threshold proportion. Aoyama et al reported that administration of nutritional support with omega- 3 fatty acids caused a $100 \%$ completion rate for adjuvant chemotherapy in gastric cancer. ${ }^{6}$ We set 0.9 as the estimated proportion for our study because BTC has about a $10 \%$ early recurrence rate.

Under an estimation of 0.76 for a threshold proportion and 0.9 for an estimated proportion, 51 participants are required for a one-sided significance level 0.025 and a power of 1-0.2. There is a potential for a few dropouts from follow-up, so we plan for a total of 55 participants. If the early recurrence rate is the same as that of a previous report $(12 \%)$ and the estimated proportion is 0.88 , the power will be 0.56 .

There are about 25 target surgical operations in our institution per year, so we set a registration period of 3 years.

\section{Study participants}

Potential participants are all patients in whom the surgical resection of BTC is performed during the study period. After surgical resection, participants are recruited and must provide written, informed consent before enrolment for the study. Participants who satisfy all the inclusion criteria and none of the exclusion criteria (box 1) are enrolled in this study.

\section{Schedule of the study}

Table 1 shows the schedule of the study. After written, informed consent, researchers perform a screening test and check inclusion and exclusion criteria. If participants meet all eligibility criteria, they are registered in the study. No protocol treatment before registration and no revocation after registration are permitted. After all data are entered and registration numbers given, then patients 


\section{Box 1 Inclusion and exclusion criteria for patients}

\section{Inclusion criteria}

1. Pathologically diagnosed as adenocarcinoma (in case of a combined cancer, adenocarcinoma is dominant).

2. Union for International Cancer Control stage is as follows: Stage I or higher in intrahepatic cholangiocarcinoma. Stage II or higher in perihilar bile duct cancer, distal bile duct cancer, gallbladder cancer and ampulla region cancer.

3. Age $20-80$ years or younger.

4. Eastern Cooperative Oncology Group performance status is 0 or 1 .

5. CT and MRI show no metastases, and moderate or less pleural effusion* or ascitest

6. Performed pancreaticoduodenectomy, hepatectomy, bile duct resection, caudal lobectomy and/or cholecystectomy, with D1 or more extensive lymphadenectomył.

7. No history of any prior chemotherapy or radiation therapy.

8. 14-70 days from surgical resection.

9. Good oral intake.

10. No watery diarrhoea.

Meets all the following laboratory data values within 14 days before registration:
a. Neutrophils $\geq 1200 / \mathrm{mm}^{3}$
b. Platelets $\geq 10000 / \mathrm{mm}^{3}$
c. Haemoglobin $\geq 8.0 \mathrm{~g} / \mathrm{L}$
d. Total bilirubin $\leq 2.0 \mathrm{mg} / \mathrm{dL}$
e. Aspartate aminotransferase $\leq 100 \mathrm{IU} / \mathrm{L}$
f. Alanine aminotransferase $\leq 100 \mathrm{IU} / \mathrm{L}$
g. Serum creatinine $\leq 1.2 \mathrm{mg} / \mathrm{dL}$
h. Creatinine clearance (CC) $\geq 40 \mathrm{~mL} / \mathrm{min}$ (CC calculated by Cockcroft-Gault Equation)

11. Written informed consent

\section{Exclusion criteria}

1. Allergic predisposition or drug hypersensitivity.

2. Double or multiple cancers diagnosed or treated in the past 5 years (except for carcinoma in situ).

3. Active infectious disease (except for virus hepatitis).

4. Fever above $38^{\circ} \mathrm{C}$.

5. Current pregnancy, breastfeeding, planning to become pregnant during the study.

6. Psychological disease and/or symptoms.

7. Severe liver or renal dysfunction.

8. Uncontrollable hypertension or diabetes mellitus.

9. Uncontrollable ischaemic heart disease:

- Developing angina or worsening within 3 weeks.

- Developing myocardial infarction within 6 months.

10. Taking flucytosine, phenytoin or warfarin potassium.

11. Interstitial pneumonitis, pulmonary fibrosis and pulmonary emphysema.

12. Taking omega-3 fatty acid formulations.

13. Considered unsuitable by their attending physician.

${ }^{*}$ Moderate plural effusion is defined as effusion occupying one third of the lung field by chest X-ray.

†Moderate ascites is defined as ascites within the pelvic cavity.

$\ddagger$ In the case of intrahepatic cholangiocarcinoma without invasion to the hepatic portal region, lymphadenectomy is not necessary.

are counted as registered. Participants undergo protocol treatment within 14 days after registration.

After registration, this study consists of a maximum 2-week preobservation period, 24-week treatment period and 4-week postobservation period. During the preobservation period, participants taking an antilipemic agent change their drug to omega-3 fatty acid formulations.

Participants do not receive any anticancer treatment after protocol treatment until the recurrence of cancer.

\section{Intervention}

S-1

Participants receive S-1 orally with the amount calculated considering body surface area and creatinine clearance (table 2). Body weight for this calculation is at registration. They receive S- 1 twice daily for 28 consecutive days, and the drug is withdrawn for the next 14 days (one cycle). This administration of S-1 is repeated every 6 weeks for up to four cycles. If a cycle ends within 42 days for some reason, participants undergo an additional cycle until the end of the 24-week treatment period. A cycle is not newly started after a treatment period, and ongoing cycles on day 168 of the treatment period continue to the end.

Participants should meet the following criteria when starting the treatment from the second cycle: (1) Eastern Cooperative Oncology Group performance status is 0,1 or 2; (2) neutrophil count is 1000 cells $/ \mu \mathrm{L}$ or higher; (3) platelet count is 50000 cells $/ \mu \mathrm{L}$ or higher; (4) aspartate aminotransferase concentration is less than three times the upper limit; (5) alanine aminotransferase concentration is less than three times the upper limit; (6) total bilirubin (T-Bil) concentration is less than 1.5 times the upper limit; (7) percutaneous arterial oxygen saturation is $90 \%$ or more, and respiratory status dose is not worse than at registration and (8) researchers consider that the treatment can be performed safely.

\section{Omega-3 fatty acid formulations}

All participants take LOTRIGA $2 \mathrm{~g}$ (Takeda Pharmaceutical Co.), which includes $2 \mathrm{~g}$ of omega-3 fatty acids from day 1 to day 168 of the treatment period.

\section{Withdrawal and reduction of the drug}

If any adverse events are observed, researchers should withdraw S-1 and omega-3 fatty acid formulations. When participants meet the criteria for restarting within 7 days, they restart S-1 as the same cycle. In this case, the next cycle can start from the scheduled day with at least a 7-day interval. When participants do not meet the criteria for restart after more than 8 days, the cycle stops at that time. If participants meet the starting criteria, they can start a new cycle. When participants do not meet the restart criteria after more than 42 days since the last day of taking S-1, they should stop the protocol treatment.

When restarting S-1 after withdrawal due to an adverse event, participants get a reduced dose of S-1. The daily dose of S-1 is reduced from 120 to $100 \mathrm{mg}$, from 100 to $80 \mathrm{mg}$, from 80 to $60 \mathrm{mg}$ or from 60 to $50 \mathrm{mg}$. Participants have two opportunities for reducing the drug. Participants taking $50 \mathrm{mg}$ of S-1 should stop the protocol treatment. 
Table 1 Schedule of enrolment, interventions and assessments

\begin{tabular}{|c|c|c|c|c|c|c|c|c|}
\hline \multirow[b]{3}{*}{ Timepoint (week) } & \multicolumn{8}{|l|}{ Study period } \\
\hline & \multirow{2}{*}{$\begin{array}{l}\text { Preobservation } \\
14 \text { days before registration }\end{array}$} & \multicolumn{6}{|c|}{ Treatment period } & \multirow{2}{*}{$\begin{array}{l}\text { Postobservation } \\
28\end{array}$} \\
\hline & & 0 & $2,4,6$ & 9 & 12 & $15,18,21$ & Close-out, 24 & \\
\hline Visit & 1 & 2 & $3,4,5$ & 6 & 7 & $8,9,10$ & 11 & 12 \\
\hline \multicolumn{9}{|l|}{ Enrolment } \\
\hline Eligibility screen & $x$ & & & & & & & \\
\hline Informed consent & $x$ & & & & & & & \\
\hline \multicolumn{9}{|l|}{ Interventions } \\
\hline \multicolumn{9}{|l|}{ S-1 } \\
\hline \multicolumn{9}{|l|}{ Lotriga } \\
\hline General status & & & & & $x$ & & $x$ & $x$ \\
\hline Blood test & & & $x$ & $x$ & $x$ & $x$ & $x$ & $x$ \\
\hline ConA, PHA, DAO & $x$ & & & & $x$ & & $x$ & \\
\hline Adverse event & & & $x$ & $x$ & $x$ & $x$ & $x$ & $x$ \\
\hline
\end{tabular}

Background includes age, gender, body weight, height, history, complication, date of diagnosis, family history, medication, and operative information (eg, date, method, blood loss and operation time). Screening tests are general status, blood test, urinary test, CT and ECG. General status includes blood pressure, body temperature, pulse rate and respiratory rate. Blood test includes complete blood count, differential count of leukocytes, total protein, albumin, total bilirubin, creatinine, aspartate transaminase, alanine aminotransferase, alkaline phosphatase, lactate dehydrogenase, $\mathrm{Na}, \mathrm{K}$, total cholesterol, high-density lipoprotein cholesterol, low-density lipoprotein cholesterol and triglyceride.

ConA, concanavalin A; DAO, diamine oxidase; PHA, phytohaemagglutinin.

If participants develop febrile neutropenia, they should be evaluated for infectious status, and antimicrobial agent or granulocyte colony stimulating factor administered.

\section{Adherence}

Researchers provide patient compliance instructions, and confirm at each visit the remnant of drugs and whether participants have ingested the correct dose of S-1.

\section{Concomitant drugs and therapy}

There are no concomitant drugs and therapy during this study.

\section{Outcomes}

\section{Primary outcome}

The primary outcome is the completion rate of adjuvant chemotherapy. Completion is defined as participants who can finish a total of four cycles of S-1 adjuvant chemotherapy, regardless of relative performance index

\begin{tabular}{lll}
\hline Table 2 & Dose of S-1 & \\
\hline \multicolumn{3}{c}{ Dose of S-1 } \\
\hline $\begin{array}{l}\text { Body surface area } \\
\left(\mathrm{m}^{2}\right)\end{array}$ & $\begin{array}{l}50 \mathrm{~mL} / \mathrm{min} \leq \mathrm{CC}<60 \mathrm{~mL} / \\
\mathrm{min}\end{array}$ & $60 \mathrm{~mL} / \mathrm{min} \leq \mathrm{CC}$ \\
Under 1.25 & $60 \mathrm{mg} /$ day & $80 \mathrm{mg} /$ day \\
$1.25-1.5$ & $80 \mathrm{mg} /$ day & $100 \mathrm{mg} /$ day \\
Over 1.5 & $100 \mathrm{mg} /$ day & $120 \mathrm{mg} /$ day \\
\hline
\end{tabular}

$\mathrm{CC}$, creatinine clearance. (proportion of actual dose per expected dose) after surgical resection of BTC.

\section{Secondary outcomes}

Secondary outcomes are as follows:

1. Postoperative cholangitis.

2. Time to recurrence or distant metastasis.

3. Changes in nutritional index from registration until the completion of chemotherapy.

4. Changes in the lymphocyte blast transformation test induced by phytohaemagglutinin and concanavalin A during adjuvant chemotherapy.

5. Change in diamine oxidase serum activity during adjuvant chemotherapy.

6. All adverse events after medication. A modified version of the Common Terminology Criteria for Adverse Events (CTCAE V.4.0) is used to grade adverse events.

Prospective adverse events are as follows: coagulation disorder, lipid metabolism disorder, abnormality of laboratory data, fatigue, fever, gastrointestinal disorders, skin and subcutaneous tissue disorders, metabolism and nutrition disorders, nervous system disorders, blood and lymphatic system disorders, eye disorders, infections and infestations, injury and procedural complications.

\section{Data analysis}

Participants who are recruited into this study and are administered one of the test drugs at least once are included in the full analysis set (FAS). Participants who 
cannot provide baseline data and have serious violations of protocol are not included in the FAS. The per protocol set (PPS) is defined as participants in the FAS who comply with exclusion criteria, concomitant drugs and concomitant therapy. An analysis of FAS is performed for each outcome. Safety is analysed in PPS. All analysis is performed with fixed data.

\section{Primary outcome}

The point estimate of the completion rate of $\mathrm{S}-1 \%$ and 95\% CI is calculated. Participants who complete S- 1 are defined as participants who have performed four scheduled cycles of S-1, or have continued treatment for 168 days.

\section{Secondary outcomes}

Analyses of secondary outcomes take the form of simple descriptive statistics (eg, proportions and IQRs, means and SDs) and where appropriate, point estimates of effect sizes (eg, mean differences) and associated 95\% CIs. Recurrence-free survival curves are calculated using the Kaplan-Meier method, and 95\% CIs are calculated.

\section{Safety analysis}

In the evaluation of the safety of the study, all adverse events due to the study are counted. Severe adverse events are defined as the following three events: grade 4 of a non-blood system disorder, early mortality within 30 days after the last treatment or mortality causally related to the protocol treatment.

Interim analysis is to be performed in this study. All statistical analyses are conducted using JMP software (SAS V.13.0).

\section{Data management}

Researchers make case report forms (CRF) for each participant. The modification of a CRF is permitted only when it does not exceed the prescribed range and is not a burden to participants. The principal investigator confirms and signs the CRF. CRFs are made at registration (within 2 weeks), during a treatment period (each two cycles), at the end of treatment (within 30 days) and in the follow-up period.

\section{Data and safety monitoring}

Independent data and safety monitoring is conducted. The following materials are reviewed every 6 months: informed consent obtained and signed, participant retention, study implementation system, study safety and data, and study progress.

\section{Confidentiality}

All study-related information is stored securely at the study site and identified by a coded number only to maintain participant confidentiality. All participant information is stored in locked file cabinets in areas with limited access. All records that contain names or other personal identifiers are stored separately from study records identified by code number. All local databases are secured with password-protected access systems. A participant's study information is not be released outside of the study without the written permission of the participant.

\section{ETHICS AND DISSEMINATION}

This study is conducted according to the declaration of Helsinki, Ethical Guidelines for Medical and Health Research Involving Human Subjects in Japan, and Management Guidelines of Conflict of Interest in our institution.

Researchers explain the study to potential participants using information sheets. Patients are given the opportunity to ask questions and have enough time to consent to the study. Researchers obtain written consent from patients willing to participate in the study. Information sheets and consent forms are provided for all participants involved in the study.

The results of this study will be disseminated through academic conferences and peer-reviewed journals. All authors will review and approve the paper before publication. If there are several papers related to this study, the investigator will appoint each author.

\section{DISCUSSION}

The biliary tract is divided into four anatomical components: intrahepatic bile duct, extrahepatic bile duct, gallbladder and ampulla of vater. Cancer staging is different for each origin, and the prognosis also differs. To select proper patients for this study, we decided that the indication criteria was stage I or higher for intrahepatic cholangiocarcinoma and stage II or higher for other BTCs according to a Japan Clinical Oncology Group 1202 trial, ${ }^{14}$ which was conducted on the basis of the following data. The 5-year survival rate in stage I from a 2008-2013 nationwide survey in Japan ${ }^{15}$ was $91 \%$ for gallbladder cancer and 92\% for ampullary region cancer; a difference compared with other digestive organ cancers in stage I was not found. Although the 5-year survival rate is somewhat low, being $74 \%$ for perihilar bile duct cancer and 78\% for distal bile duct cancer, cancer deaths for these diseases are not common. Potential reasons for the low ratios include perioperative deaths and late complications such as cholangitis. Therefore, since we had regarded that these patients get few benefits from adjuvant chemotherapy, in principle adjuvant chemotherapy was not performed for stage I. In comparison, the 5-year survival rate of intrahepatic cholangiocarcinoma in patients with the tumour, less than $2 \mathrm{~cm}$ in diameter, that corresponds to stage I is $36.3 \%$ in Japan's National Primary Liver Cancer Follow-up Survey (the 18th), which was worse than the same stage for other BTCs. Therefore, adjuvant chemotherapy seems to be beneficial for patients of stage I intrahepatic cholangiocarcinoma.

Though the target for adjuvant chemotherapy for BTC is still controversial, we have selected the relatively poor-prognosis group in BTC. Moreover, to align 
the patients postsurgical background, we have selected the type of surgical operation, which includes biliary reconstruction.

The most common reason for the interruption of adjuvant chemotherapy was an adverse event in BTC. ${ }^{3}$ Seo et $a l^{16}$ reported that hypoalbuminaemia $(<3.5 \mathrm{mg} / \mathrm{dL})$ was associated with adverse events greater than grade 3 in gastric cancer. Hypoalbuminaemia is also a risk factor for the interruption of adjuvant chemotherapy in patients with pancreatic cancer. ${ }^{17}$ The albumin level is one of the indications of nutritional status. According to a previous study, body weight loss is associated with the continuance of adjuvant chemotherapy, ${ }^{6}$ and nutritional intervention, including omega-3 fatty acids, has an effect on several cancers. ${ }^{78}$

It is well known that chemotherapy including 5-fluorouracil (5-FU) causes gastrointestinal injury. ${ }^{18} 5$-FU also showed toxicity to helper $\mathrm{T}$ cells in mice, which seemed to cause immunosuppression. ${ }^{19}$ These changes may result in $\mathrm{BT} .{ }^{20}$ From the viewpoint of immunity and infection, omega-3 fatty acids play a preventive role. Matsunaga et al reported that a diet containing omega-3 fatty acid suppressed the thickening of mucosa, submucosa and the muscular layer due to inflammation in mice compared with a control diet. ${ }^{21}$ Moreover, omega-3 fatty acids inhibit infiltration of inflammatory cells into the muscularis mucosa. ${ }^{19}$ In addition, the rate of BT and ileal change in preoperative and postoperative feeding with omega-3 fatty acids in rats with a common bile duct ligation were the same as that of the control group, while BT and atrophy of intestinal mucosa in the postoperative feeding group increased compared with the control group. ${ }^{22}$ From these reports, it is thought that the administration of omega-3 fatty acids from the start of chemotherapy, when intestinal mucosal disorder is not yet occurring, can keep the intestinal mucosa normal and prevent intestinal inflammation and the interruption of anticancer agents by infection.

In addition to the nutritional aspect, omega-3 fatty acids have an anti-inflammatory effect. After biliary reconstruction, it is easy for patients to experience cholangitis and stop chemotherapy. Omega-3 fatty acids are known to calm acute inflammation by regulating inflammatory cytokines. ${ }^{9}$ Furthermore, in vivo experiments showed suppression of the proliferation of cancer cells by inducing apoptosis in several cancers. ${ }^{10} 1213$

In this study, we expect that omega-3 fatty acids will improve the completion rate of adjuvant chemotherapy and lead to a good prognosis in patients with BTC. However, this study may not be generalisable to other chemotherapy regimens since only S-1 is the target as adjuvant chemotherapy.

Acknowledgements I would like to thank Dr S Murakami and Kobe University Hospital Clinical and Translational Research Center for the support in setting up this research and preparing the manuscript.

Contributors $\mathrm{KU}$ designed the study and wrote the initial draft of the manuscript. TA and TF contributed to analysis and interpretation of data, and assisted in the preparation of the manuscript. DT, MA and YH contributed to analysis and interpretation of data. MA, HM, SK, KK, ST, MT, HT and MK contributed to collect the clinical data. All authors approved the final version of the manuscript and agreed to be accountable for all aspects of the work in ensuring that questions related to the accuracy or integrity of any part of the work are appropriately investigated and resolved.

Funding The authors have not declared a specific grant for this research from any funding agency in the public, commercial or not-for-profit sectors.

Competing interests None declared.

Patient consent for publication Not required.

Ethics approval The protocol has been approved by the Institutional Review Board of Kobe University Hospital (№ 290086).

Provenance and peer review Not commissioned; externally peer reviewed.

Open access This is an open access article distributed in accordance with the Creative Commons Attribution Non Commercial (CC BY-NC 4.0) license, which permits others to distribute, remix, adapt, build upon this work non-commercially, and license their derivative works on different terms, provided the original work is properly cited, appropriate credit is given, any changes made indicated, and the use is non-commercial. See: http://creativecommons.org/licenses/by-nc/4.0/.

\section{REFERENCES}

1. Miyakawa S, Ishihara S, Horiguchi A, et al. Biliary tract cancer treatment: 5,584 results from the biliary tract cancer statistics registry from 1998 to 2004 in Japan. J Hepatobiliary Pancreat Surg 2009;16:1-7.

2. Waseem D, Tushar P. Intrahepatic, perihilar and distal cholangiocarcinoma: management and outcomes. Ann Hepatol 2017:16:133-9.

3. Nakachi K, Konishi M, Ikeda M, et al. Feasibility study of postoperative adjuvant chemotherapy with S-1 in patients with biliary tract cancer. Int J Clin Oncol 2018;23:894-9.

4. Sakuramoto S, Sasako M, Yamaguchi T, et al. Adjuvant chemotherapy for gastric cancer with $\mathrm{S}-1$, an oral fluoropyrimidine. $N$ Engl J Med 2007;357:1810-20.

5. Uesaka K, Boku N, Fukutomi A, et al. Adjuvant chemotherapy of S-1 versus gemcitabine for resected pancreatic cancer: a phase 3 , open-label, randomised, non-inferiority trial (JASPAC 01). Lancet 2016;388:248-57.

6. Aoyama T, Yoshikawa T, Shirai J, et al. Body weight loss after surgery is an independent risk factor for continuation of S-1 adjuvant chemotherapy for gastric cancer. Ann Surg Oncol 2013;20:2000-6.

7. Aoyama T, Hayashi T, Fujikawa $\mathrm{H}$, et al. [Effect of enteral nutrition enriched with eicosapentaenoic acid on body weight loss and compliance with S-1 adjuvant chemotherapy after gastric cancer surgery]. Gan To Kagaku Ryoho 2013;40:2289-91.

8. Murphy RA, Mourtzakis M, Chu QSC, et al. Nutritional intervention with fish oil provides a benefit over standard of care for weight and skeletal muscle mass in patients with nonsmall cell lung cancer receiving chemotherapy. Cancer 2011;117:1775-82.

9. Serhan CN. Pro-Resolving lipid mediators are leads for resolution physiology. Nature 2014;510:92-101.

10. Cao W, Ma Z, Rasenick MM, et al. N-3 poly-unsaturated fatty acids shift estrogen signaling to inhibit human breast cancer cell growth. PLoS One 2012;7:e52838.

11. Chagas TR, Borges DS, de Oliveira PF, et al. Oral fish oil positively influences nutritional-inflammatory risk in patients with haematological malignancies during chemotherapy with an impact on long-term survival: a randomised clinical trial. J Hum Nutr Diet 2017;30:681-92.

12. Mizoguchi $\mathrm{K}$, Ishiguro $\mathrm{H}$, Kimura $\mathrm{M}$, et al. Induction of apoptosis by eicosapentaenoic acid in esophageal squamous cell carcinoma. Anticancer Res 2014;34:7145-9.

13. Shirota T, Haji S, Yamasaki M, et al. Apoptosis in human pancreatic cancer cells induced by eicosapentaenoic acid. Nutrition 2005;21:1010-7.

14. Nakachi K, Konishi M, Ikeda M, et al. A randomized phase III trial of adjuvant S-1 therapy vs. observation alone in resected biliary tract cancer: Japan clinical Oncology Group study (JCOG1202, Ascot). Jpn J Clin Oncol 2018;48:392-5.

15. Ishihara S, Horiguchi A, Miyakawa S, et al. Biliary tract cancer registry in Japan from 2008 to 2013. J Hepatobiliary Pancreat Sci 2016;23:149-57. 
16. Seo SH, Kim S-E, Kang Y-K, et al. Association of nutritional statusrelated indices and chemotherapy-induced adverse events in gastric cancer patients. BMC Cancer 2016;16:900.

17. Matsumoto I, Tanaka M, Shirakawa S, et al. Postoperative serum albumin level is a marker of incomplete adjuvant chemotherapy in patients with pancreatic ductal adenocarcinoma. Ann Surg Oncol 2015;22:2408-15

18. Murakami M, Sato N, Tashiro K, et al. Effects of caloric intake on intestinal mucosal morphology and immune cells in rats treated with 5-fluorouracil. J Clin Biochem Nutr 2009;45:74-81.

19. Merluzzi VJ, Last-Barney K, Susskind BM, et al. Recovery of humoral and cellular immunity by soluble mediators after 5 -fluorouracilinduced immunosuppression. Clin Exp Immunol 1982;50:318-26.
20. Tsuji E, Hiki N, Nomura S, et al. Simultaneous onset of acute inflammatory response, sepsis-like symptoms and intestinal mucosal injury after cancer chemotherapy. Int $\mathrm{J}$ Cancer 2003;107:303-8.

21. Matsunaga $\mathrm{H}$, Hokari $\mathrm{R}$, Kurihara $\mathrm{C}$, et al. Omega-3 polyunsaturated fatty acids ameliorate the severity of ileitis in the senescence accelerated mice (SAM)P1/Yit mice model. Clin Exp Immunol 2009;158:325-33.

22. Zulfikaroglu B, Zulfikaroglu E, Ozmen MM, et al. The effect of immunonutrition on bacterial translocation, and intestinal villus atrophy in experimental obstructive jaundice. Clin Nutr 2003;22:277-81. 